\title{
Effect of atrial septostomy on the survival of patients with severe pulmonary arterial hypertension
}

\author{
J. Sandoval, J. Gaspar, H. Peña, L.E. Santos, J. Córdova, K. del Valle, \\ A. Rodríguez and T. Pulido
}

ABSTRACT: Atrial septostomy (AS) is a palliative treatment for right ventricular failure from severe pulmonary arterial hypertension (PAH). We sought to investigate the effect of AS, alone or combined with PAH-specific pharmacotherapy, on the survival of patients with PAH.

We performed a retrospective analysis of the functional and haemodynamic changes in patients with PAH following AS, and long-term survival characteristics for the whole group and separately for the subgroup who received post-procedural pharmacotherapy.

50 procedures performed in 34 patients (mean \pm SD age $35 \pm 10$ yrs) resulted in haemodynamic and symptomatic improvement in most of the patients. Only one (2\%) procedure-related death occurred. Due to spontaneous closure of the defect, AS was repeated in 10 patients. In 21 patients, AS was the only form of treatment, while 11 received additional pharmacotherapy after AS. During follow-up (58.5 \pm 38 months), 21 patients died; median survival of the group was 60 months (95\% Cl 43-77 months). Median survival for patients on pharmacotherapy additional to AS was 83 months (95\% Cl 57-109 months), which was better than that for patients with AS alone (53 months, 95\% Cl 39-67 months) (log-rank 6.52; $p=0.010$ ).

In selected patients with $\mathrm{PAH}, \mathrm{AS}$ is a safe and effective intervention that exerts a beneficial impact on long-term survival. Survival appears to be improved when AS is combined with PAHspecific pharmacotherapy.

KEYWORDS: Atrial septostomy, pulmonary hypertension, survival

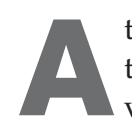

trial septostomy (AS) represents an additional strategy for the treatment of right ventricular failure (RVF) from severe pulmonary arterial hypertension (PAH) [1]. Several reasons still justify its use: 1 ) the deleterious impact of RVF on patient survival [2, 3]; 2) the unpredictable response to medical treatment; and 3) the disparity in the availability of these treatments throughout the world and the limited access to lung transplantation [4].

Little information exists regarding the long-term impact of AS on the survival of PAH patients [5-7]. In most of the reported series [5, 7-11], the procedure was performed after all available medical therapy failed, late in the course of the disease, when the risk of procedural death is increased and the impact of the intervention on long-term survival is minimised. We describe the effect of AS on the survival of a group of patients with PAH in whom the procedure was performed either as an isolated treatment intervention or prior to the use of PAH-specific pharmacological therapy.

\section{METHODS}

\section{Patient population}

Between January 1995 and November 2006, we performed 50 AS procedures in 34 patients with $\mathrm{PAH}$, including 15 case that have been previously reported [12]. All patients underwent thorough diagnostic workup that included: history and physical examination; haematology, chemistry and coagulation studies; pulmonary and liver function tests; chest radiography and computed tomography evaluation; and echocardiography and right heart catheterisation. The diagnosis of $\mathrm{PAH}$ was established by exclusion of secondary causes of pulmonary hypertension and demonstration of a mean pulmonary artery pressure $\left(\bar{P}_{\mathrm{pa}}\right)$ of $>25$ $\mathrm{mmHg}$ at rest, and a pulmonary capillary wedge pressure $(P \mathrm{pcw})$ of $<15 \mathrm{mmHg}$ [13].

\section{Procedure}

AS for the treatment of PAH has been approved by our institutional ethics committee (Instituto Nacional de Cardiología Ignacio Chávez, Mexico City, Mexico). Written consent was obtained after

\section{AFFILIATIONS}

Cardiopulmonary and Interventional Cardiology Depts, Instituto Nacional de Cardiología Ignacio Chávez,

Mexico City, Mexico.

\section{CORRESPONDENCE}

J. Sandoval

Cardiopulmonary Dept

Instituto Nacional de Cardiología

Ignacio Chávez

Juan Badiano No. 1

Colonia Sección XVI

Tlalpan

14080 México

DF México

Mexico

E-mail: julio.sandoval@

cardiologia.org.mx

Received:

May 072010

Accepted after revision:

Feb 082011

First published online:

Feb 242011 
an unbiased presentation of the palliative nature of the procedure, and its risks and expected outcome was presented to each patient.

Our technique for balloon dilation AS has been reported elsewhere [12, 14]. Briefly, baseline right and left heart pressures were recorded simultaneously and cardiac output was calculated by the Fick method. Following trans-septal puncture using standard technique, the septostomy orifice was balloon-dilated in a carefully graded step-by-step approach, beginning at a diameter of $4 \mathrm{~mm}$, and followed by 8-, 12- and 16-mm dilatations. Between each step and after a 3-min waiting period allowed for stability, left ventricular end-diastolic pressure (LVEDP) recordings and arterial oxygen saturation $\left(\mathrm{Sa}_{\mathrm{a}} \mathrm{O}_{2}\right)$ were obtained. The final size of the defect was individualised in each patient and correlated with the time at which any of the following first occurred: 1) an LVEDP increase of $>18 \mathrm{mmHg}$; 2) a $\mathrm{Sa}_{1} \mathrm{O}_{2}$ reduction of $\sim 80 \%$; or 3 ) a $10 \% \mathrm{Sa}_{\mathrm{O}_{2}}$ decrease from baseline.

Follow-up of the patients was performed in the intensive care area for the first $48 \mathrm{~h}$, where continuous supplementary oxygen was administrated. All patients were followed at our outpatient clinic with particular care to maintain correct oral anticoagulation and appropriate haemoglobin levels. PAH-specific pharmacological treatment for some of the patients was dictated by the availability of these drugs at our centre. Long-term follow-up information was obtained during outpatient hospital visits, or by telephone calls to the patients or their relatives. In the case of death, all efforts were made to establish cause.

\section{Data analysis}

We analysed the clinical, functional and haemodynamic variables before and after the procedure. We also analysed the long-term survival characteristics for the whole group and separately in the subgroup of patients who received PAHspecific pharmacological treatment after the procedure. The Kaplan-Meier method was used to estimate overall survival distribution. The date of the first AS was used as the index from which to determine survival. Differences in survival curves for patients with and without concomitant pharmacological treatment were tested by the log-rank procedure. Univariate analysis based on the Cox proportional hazards model was used to examine the relationship between survival and selected demographic, medical history, laboratory and haemodynamic variables. Data are presented as hazard ratios with $95 \%$ confidence intervals. For all other analyses, we used paired and unpaired t-tests. Data are presented as means and standard deviations. A p-value $<0.05$ was considered significant in all comparative analyses. Analyses were performed using the SPSS package for Windows, version 10.0 (IBM, Somers, NY, USA).

\section{RESULTS}

\section{Patients}

Most patients were female $(85 \%)$, with a mean \pm SD age of $35 \pm 10$ yrs (range 22-62 yrs). Aetiological diagnoses included idiopathic PAH in 29 (85\%) patients, distal (inoperable) chronic thromboembolic pulmonary hypertension in three (9\%), PAHassociated with lupus in one (3\%) and PAH associated with a previously corrected ventricular septal defect in another (3\%). All patients had severe pulmonary hypertension, with a threefold increase in $\bar{P}_{\text {pa }}(70 \pm 14 \mathrm{mmHg})$, a normal $P_{\text {pcw }}(8 \pm 5 \mathrm{mmHg})$ and variable degrees of right heart failure (RHF), as reflected by a cardiac index of $2.32 \pm 0.8 \mathrm{~L} \cdot \mathrm{min} \cdot \mathrm{m}^{-2}$ and an increased right atrial pressure (Pra) of $12 \pm 5.5 \mathrm{mmHg}$.

Most patients had severe functional limitation. World Health Organization (WHO) functional class was 3.5 $\pm 0.6 ; 18(53 \%)$ patients were in functional class IV, $14(41 \%)$ were in class III and two $(6 \%)$ were in class II. Exercise endurance, as assessed by the 6-min walk test $(6 \mathrm{MWT})$, was $100 \pm 114 \mathrm{~m}$ (range 0-380 m). During diagnostic right heart catheterisation, none of these patients had responded to acute vasodilator challenge. The symptomatic indications for AS were syncope in nine $(26 \%)$ patients, RHF in $14(41 \%)$ and the coexistence of both in $11(32 \%)$.

\section{Immediate outcome}

One of the patients (previously reported) died $48 \mathrm{~h}$ after the procedure as a result of refractory hypoxaemia. Most $(88 \%)$ of the 33 surviving patients had clinical improvement after AS and four $(12 \%)$ remained unchanged. The functional and haemodynamic results before and after AS are shown in table 1. The mean final size of the balloon used for initial septostomy was $8.5 \pm 2.5 \mathrm{~mm}$ (range 6-16 $\mathrm{mm}$ ). After the procedure there was a moderate but significant decrease in right ventricular enddiastolic pressure (RVEDP), a significant increase in cardiac index and, as expected, a significant decrease in $\mathrm{Sa}_{1} \mathrm{O}_{2}$. WHO functional class improved and the $6 \mathrm{MWT}$, reassessed at 2 weeks to 1 month after the procedure, increased significantly following AS. The change in cardiac index correlated with the change in 6MWT $(\mathrm{r}=0.66 ; \mathrm{p}<0.017)$ and the $6 \mathrm{MWT}$ after septostomy correlated with survival $(\mathrm{r}=0.58 ; \mathrm{p}<0.016)$.

Except for the conventional use of diuretics, digitalis and anticoagulants, which was common to all patients, in 21 of the surviving patients, AS was as the only form of treatment. 11 of the patients received $\mathrm{PAH}$-specific pharmacological treatment at different time intervals after AS (18 \pm 13 months); most of them $(82 \%)$ within the first 2 yrs. The medications included subcutaneous treprostinil $(n=5)$, bosentan $(n=3)$, sitaxsentan $(n=1)$, sildenafil $(n=1)$ and oral treprostinil $(n=1)$. In one of the patients, septostomy was performed after the failure of pharmacological treatment with subcutaneous treprostinil, which she had received for 6 yrs. For the purpose of the present study, this patient was excluded from the survival analysis.

\begin{tabular}{|c|c|c|c|}
\hline Variable & Before AS & After AS & p-value \\
\hline RVEDP $\mathrm{mmHg}$ & $14.5 \pm 6$ & $10.7 \pm 5.9$ & 0.001 \\
\hline LVEDP $\mathrm{mmHg}$ & $6.4 \pm 3.4$ & $8.8 \pm 2.7$ & 0.001 \\
\hline $\bar{P}$ pa $\mathrm{mmHg}$ & $66 \pm 13$ & $61 \pm 16$ & 0.002 \\
\hline Cardiac index $\mathrm{L} \cdot \mathrm{min} \cdot \mathrm{m}^{-2}$ & $2.26 \pm 0.43$ & $2.97 \pm 0.83$ & 0.001 \\
\hline $\mathrm{Sa}, \mathrm{O}_{2} \%$ & $91.7 \pm 3.8$ & $84 \pm 6.3$ & 0.001 \\
\hline 6MWT m & $106 \pm 115$ & $214 \pm 99$ & 0.001 \\
\hline WHO FC & $3.47 \pm 0.62$ & $2.19 \pm 0.54$ & 0.001 \\
\hline
\end{tabular}

RVEDP: right ventricular end-diastolic pressure; LVEDP: left ventricular enddiastolic pressure; $\bar{P}$ pa: mean pulmonary artery pressure; $\mathrm{Sa}_{2} \mathrm{O}_{2}$ : arterial oxygen saturation; 6MWT: 6-min walk test; WHO: World Health Organization; FC: functional class. 
AS had to be repeated in $10(29.4 \%)$ patients at different intervals due to spontaneous closure of the defect. Two patients had four septostomies, two patients had three and six had two. Repeat procedures were equally distributed between the groups with and without concomitant pharmacological treatment. In the group of patients with pharmacological treatment, there was no need for repeat procedures once the specific drug treatment was initiated.

\section{Long-term survival}

Over the whole follow-up period $(58.5 \pm 38$ months; range 3138 months), 21 (66\%) patients died, four patients were lost to follow-up at $6,15,37$ and 74 months, and seven patients survived. The time interval from procedure to death was $56 \pm 30$ months (range 3-110 months). The identified causes of death were progression of RHF $(n=16)$, cerebrovascular accident $(n=2)$, pneumonia $(n=2)$ and sudden death $(n=1)$. No post mortem studies were performed.

\section{Mortality-associated factors}

Mortality-associated factors are shown in table 2.

In the exploratory analysis of the potential factors associated with survival after the procedure, mortality was not associated with age, haemodynamic variables or AS size. A higher functional class after AS was associated with a significant risk of death, whereas higher $\mathrm{Sa}_{\mathrm{a}} \mathrm{O}_{2}$, exercise endurance and the use of specific pharmacological treatment after septostomy had a protective effect against such a risk.

Lifetime cumulative survival rates for patients with idiopathic $\mathrm{PAH}(\mathrm{n}=27)$ are shown in figure 1a. The median survival of the group was 60 months (95\% CI 41.2-78.7 months). As noted, survival after septostomy is better than the 1-, 2- and 3-yr survival rates of $65.2,52.3$ and $42.8 \%$, respectively, as predicted by the equation developed from the National Institutes of Health primary pulmonary hypertension registry data [2, 3].

The 11 patients who received PAH-specific pharmacological treatment after AS were comparable to those who had AS alone in terms of age, AS size and haemodynamic values before and after the procedure (table 3 ). Their survival, however, was better than that for patients with AS only (median survival 83 months (95\% CI 57-109 months) versus 53 months (95\% CI 39-67 months), respectively; Chi-squared log-rank 6.52; $p=0.01$; fig. $1 b$ ).

\section{DISCUSSION}

In this relatively large series of patients with $\mathrm{PAH}$, the combination of AS with PAH-specific pharmacological therapy appeared to exert a beneficial impact on long-term survival, which was superior to that provided by AS alone.

One possible explanation for this finding is the timing of performance of AS. In most reported series [5, 7-11], AS has been performed only after the failure of different $\mathrm{PAH}$-specific pharmacological interventions. In the present study, patients with concomitant pharmacological treatment had AS performed before pharmacological treatment was initiated. Although not statistically significant, the prevalence of WHO class IV patients was lower (36\%) in the AS plus PAH-specific pharmacological therapy group compared with that in the group with AS alone (62\%).

\section{Immediate outcome}

AS produced immediate haemodynamic and functional improvement in most of our patients with results that were comparable to those described in similar series [5, 7-11, 12, 1518]. The beneficial functional effects after AS appear to be related to the increase in cardiac index after septostomy [8]. In our study, the increase in 6MWT was significantly related to the increase in cardiac index after AS.

The present study also demonstrates that safety in the performance of AS is an attainable goal. Our procedure-related mortality (death that occurs during or within 1 month after the procedure) was only $2 \%$ (one death out of 50 procedures). Operator experience and strict adherence to WHO safety recommendations [6, 19] certainly account for this lower procedure-related fatality rate. In this regard, balloon dilation AS with a graded step-by-step protocol was used in most of the recent series, which also reported a procedural mortality that appeared to decrease with this technical approach [20]. Other factors, such as the timing of the procedure, may also be important. In the analysis of the collective worldwide experience with AS $[6,20]$ and other reports $[7,21]$, a mean $P_{\text {ra }}\left(\bar{P}_{\text {ra }}\right)$

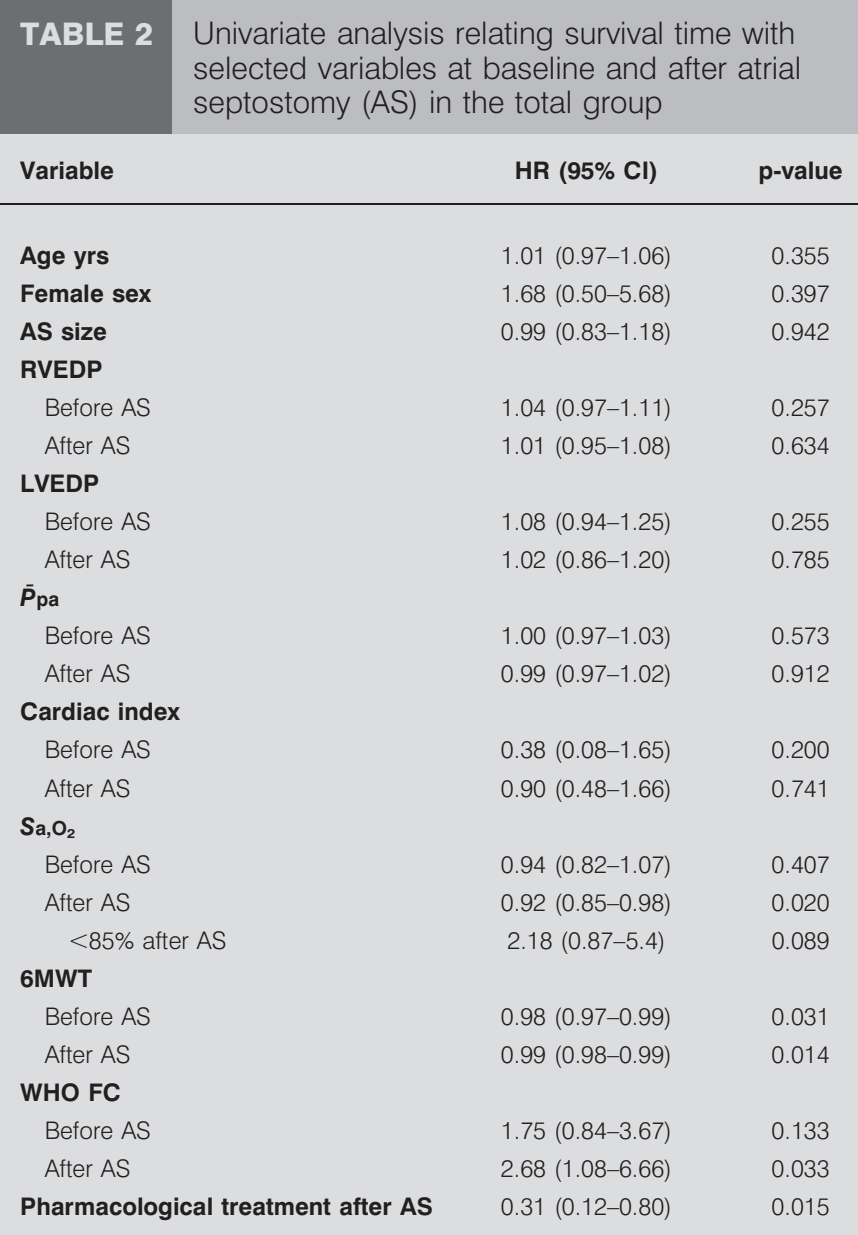

HR: hazard ratio; RVEDP: right ventricular end-diastolic pressure; LVEDP: left ventricular end-diastolic pressure; $\bar{P}$ pa: mean pulmonary artery pressure; $\mathrm{Sa}_{1} \mathrm{O}_{2}$ : arterial oxygen saturation; 6MWT: 6-min walk test; WHO: World Health Organization; FC: functional class. 

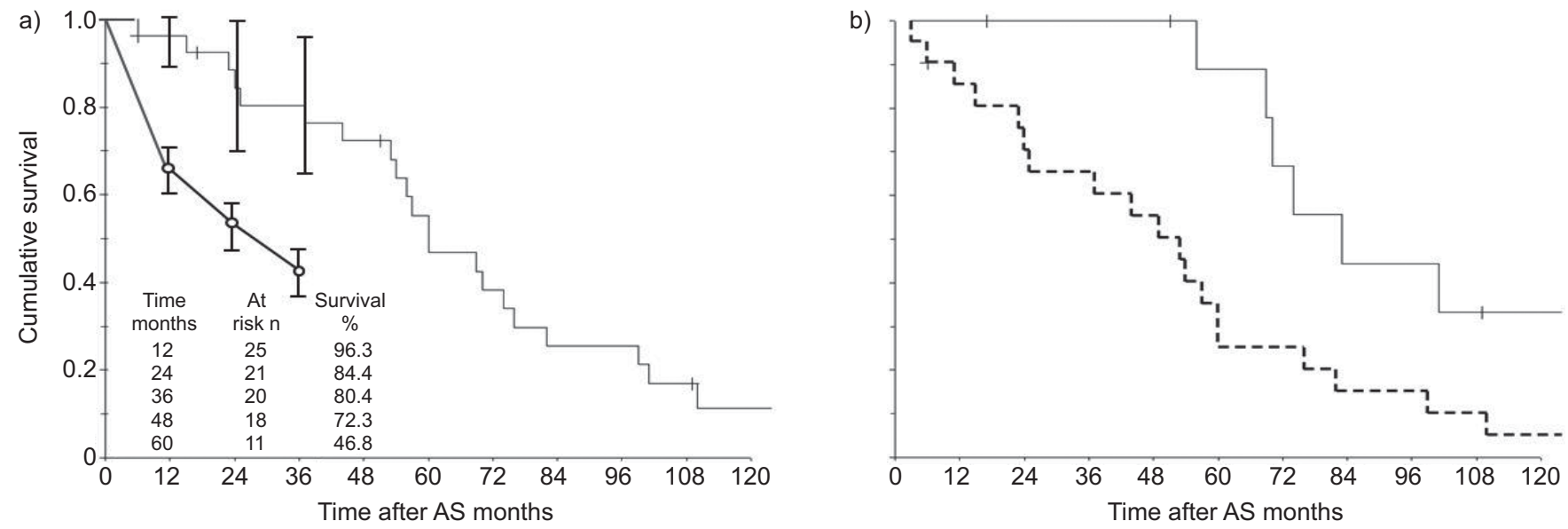

FIGURE 1. Survival after atrial septostomy (AS). a) Kaplan-Meier survival estimates after AS in the whole group of patients with pulmonary arterial hypertension (PAH). The median \pm SEM survival of the group was $60 \pm 8$ months (95\% Cl 43-77 months). A predicted survival curve $(O)$ is plotted for comparison. Error bars indicate $95 \%$ confidence intervals. b) The survival estimates for PAH patients with AS plus PAH-specific pharmacological treatment (-) were better than those in patients with AS alone (---) (median survival 83 months (95\% Cl 57-109 months) versus 53 months (95\% Cl 39-67 months), respectively; Chi-squared log-rank 6.52; $p=0.01$ ).

\begin{tabular}{|c|c|c|c|}
\hline \multirow{2}{*}{$\begin{array}{l}\text { TABLE } 3 \\
\text { Variable }\end{array}$} & \multicolumn{3}{|c|}{$\begin{array}{l}\text { Demographic, functional and haemodynamic } \\
\text { characteristics of atrial septostomy (AS) patients } \\
\text { with and without concomitant pharmacological } \\
\text { treatment }\end{array}$} \\
\hline & AS plus drugs & AS only & $\mathrm{p}$-value \\
\hline Subjects $\mathrm{n}$ & 11 & 21 & \\
\hline Females \% & 82 & 86 & 0.782 \\
\hline Age yrs & $37 \pm 9$ & $33.5 \pm 10$ & 0.339 \\
\hline \multicolumn{4}{|l|}{ WHO FC } \\
\hline Before AS & $3.27 \pm 0.65$ & $3.57 \pm 0.60$ & 0.201 \\
\hline After AS & $1.91 \pm 0.30$ & $2.33 \pm 0.58$ & 0.010 \\
\hline \multicolumn{4}{|l|}{ 6MWT m } \\
\hline Before AS & $194 \pm 153$ & $60 \pm 50$ & 0.082 \\
\hline After AS & $290 \pm 95$ & $174 \pm 77$ & 0.016 \\
\hline AS size $\mathrm{mm}$ & $8.0 \pm 1.5$ & $9 \pm 2.8$ & 0.202 \\
\hline \multicolumn{4}{|c|}{ RVEDP $\mathrm{mmHg}$} \\
\hline Before AS & $18 \pm 6$ & $13 \pm 4$ & 0.067 \\
\hline After AS & $13 \pm 7$ & $9.0 \pm 4.6$ & 0.096 \\
\hline \multicolumn{4}{|l|}{ LVEDP $\mathrm{mmHg}$} \\
\hline Before AS & $6.6 \pm 3$ & $5.9 \pm 4$ & 0.534 \\
\hline After AS & $10 \pm 3$ & $8.0 \pm 2.5$ & 0.071 \\
\hline \multicolumn{4}{|l|}{$\bar{P}_{\text {pa }} \mathrm{mmHg}$} \\
\hline Before AS & $67 \pm 16$ & $66 \pm 12$ & 0.856 \\
\hline After AS & $63 \pm 21$ & $59 \pm 12$ & 0.618 \\
\hline \multicolumn{4}{|c|}{ Cardiac index $L \cdot \min \cdot \mathrm{m}^{-2}$} \\
\hline Before AS & $2.53 \pm 0.71$ & $2.23 \pm 0.82$ & 0.764 \\
\hline After AS & $2.75 \pm 0.41$ & $3.1 \pm 0.96$ & 0.291 \\
\hline \multicolumn{4}{|l|}{$\mathrm{Sa}, \mathrm{O}_{2} \%$} \\
\hline Before AS & $92 \pm 3$ & $91 \pm 4$ & 0.596 \\
\hline After AS & $86 \pm 4.5$ & $83 \pm 7$ & 0.224 \\
\hline
\end{tabular}

Data are presented as mean $\pm \mathrm{SD}$, unless otherwise stated. WHO: World Health Organization; FC: functional class; 6MWT: 6-min walk test; RVEDP: right ventricular end-diastolic pressure; LVEDP: left ventricular end-diastolic pressure; $\bar{P}$ pa: mean pulmonary artery pressure; $\mathrm{Sa}_{1} \mathrm{O}_{2}$ : arterial oxygen saturation. of $>20 \mathrm{mmHg}$, a reflection of advanced disease, has been repeatedly identified as the most significant risk factor associated with procedure-related mortality. In a recent communication, MicheLETTI et al. [16] reported no fatalities associated with the procedure in 20 children with PAH. In their series, AS was performed at a relative early stage of the disease ( $\bar{P}_{\text {ra }}$ before the procedure was $9 \pm 5 \mathrm{mmHg}$ and syncope, rather than overt RHF, was the main indication for the procedure). Likewise, in our study, a significant proportion of patients $(28 \%)$ had a RVEDP of $<10 \mathrm{mmHg}$ before the procedure. Accordingly, performing AS at earlier stages of the disease may reduce the risk of death during the procedure [16].

\section{Spontaneous closure of AS}

As already described in most of the reported series, subsequent closure of the defect was a relatively frequent feature in our series. The reason for this remains unknown. In our study, there was no significant difference in age, haemodynamic profile or AS size between patients with spontaneous closure and those in whom the AS remained open. In our study, AS size refers to the maximal size of the balloon used. An echocardiography measurement of the interatrial orifice diameter was not obtained in all patients. Due to tissue elastic recoil, the true size of the defect should be smaller than the maximal balloon diameter used.

To solve the problem of closure, we elected to repeat AS as many times as necessary and achieved this without complications. Recently, this problem has been approached differently by other investigators. MicheletTI et al. [16] placed a custommade fenestrated atrial septal device at the end of the procedure to maintain the AS open. By doing this, in seven out of 20 children, the short-term spontaneous closure of the defect was successfully avoided. This approach has been followed by other investigators [22-27]. Both a high long-term occlusion rate [25] and long-term patency of these devices has been reported [26, 27]. At present, it is difficult to anticipate the long-term risk/benefit of this approach. 


\section{Effect of AS on long-term survival}

Our study series provides the longest follow-up of patients with AS in the setting of PAH and, as in other study series [5, 7, 27], AS significantly improved long-term survival in these patients. AS improved haemodynamic parameters that correlate with survival (cardiac index and right ventricular filling pressures) and 6MWT, another surrogate of survival in $\mathrm{PAH}$, and this may explain the impact of the procedure on shortterm survival. The decrease in survival after 3-5 yrs in our patients with AS alone (fig. 1b) confirms the palliative nature of this intervention [5].

To our knowledge, the present study is the first to suggest the potential benefit of combining strategies (interventional plus pharmacological) in the management of PAH. In effect, we found that the survival benefit of AS alone was significantly increased by the concomitant addition of PAH-specific drug therapies. As shown in figure 1b, the 3- and 5-yr survival of $100 \%$ and almost $90 \%$, respectively, for this combination is impressive. It is important to stress, however, that we did not include a group of patients with PAH-specific pharmacological therapy alone for comparison and, thus, we do not know whether introduction of PAH-targeted medical therapy alone would not result in identical outcome to that created by AS combined with pharmacotherapy.

Although the concomitant use of drugs and AS has been used in many of the previously reported series, the sequence of combining these strategies is different in our study. We did not wait until the failure of medical treatment was evident to perform AS; instead, AS was performed first and then the specific drugs were added when they became available. In previous reports, AS was used as the last or almost last resort in the management strategy, when the patients were most likely in an advanced stage of the disease [28]. The performance of AS at a relatively early stage of the disease in our study may probably account for the encouraging results.

\section{Limitations}

The first limitation of our study is that it was a single-centre study and the numbers of patients were limited, but this is related to the low incidence of the underlying pathology and the current availability of specific PAH therapy. Secondly, although the data were collected from an accurate follow-up database, the study design remains retrospective. Finally, as discussed, an obvious limitation of the trial is that it does not provide an answer to the question of whether the introduction of targeted therapy alone would not have resulted in identical outcome to that of AS combined with pharmacotherapy. The true benefit of an intervention can only be assessed in a randomised controlled study; however, the malignant nature of $\mathrm{PAH}$ and the availability of new specific drugs and transplantation would make it unethical to run a study with a true survival end-point.

\section{Conclusions}

In our experience, AS is a safe and effective intervention. Operator experience and strict adherence to WHO recommendations account for our low fatality rate. AS improves haemodynamic parameters that correlate with functional improvement and survival. The potential benefit of an early combination of AS and PAH-specific drug therapies suggested by the results of the present study is appealing and, in our opinion, worthy of further evaluation.

\section{STATEMENT OF INTEREST}

None declared.

\section{REFERENCES}

1 Klepetko W, Mayer E, Sandoval J, et al. Interventional and surgical modalities of treatment for pulmonary arterial hypertension. J Am Coll Cardiol 2004; 43: Suppl. 12, 73S-80S.

2 D'Alonso GE, Barst RJ, Ayres SM, et al. Survival in patients with primary pulmonary hypertension. Results of a national prospective study. Ann Intern Med 1991; 115: 343-349.

3 Sandoval J, Bauerle O, Palomar A, et al. Survival in primary pulmonary hypertension. Validation of a prognostic equation. Circulation 1994; 89: 1733-1744.

4 Tapson V. Atrial septostomy. Why we still need it. Chest 2007; 131: 947-948.

5 Kerstein D, Levy PS, Hsu DT, et al. Blade balloon atrial septostomy in patients with severe primary pulmonary hypertension. Circulation 1995; 91: 2028-2035.

6 Sandoval J, Rothman A, Pulido T. Atrial septostomy for pulmonary hypertension. Clin Chest Med 2001; 22: 547-560.

7 Law MA, Grifka RG, Mullins CE, et al. Atrial septostomy improves survival in select patients with pulmonary hypertension. Am Heart J 2007; 153: 779-784.

8 Rothman A, Slansky MS, Lucas VW, et al. Atrial septostomy as a bridge to lung transplantation in patients with severe pulmonary hypertension. Am J Cardiol 1999; 84: 682-686.

9 Reichenberger F, Pepke-Zaba J, McNeil K, et al. Atrial septostomy in the treatment of severe pulmonary arterial hypertension. Thorax 2003; 58: 797-800.

10 Kothari SS, Yusuf A, Juneja R, et al. Graded balloon atrial septostomy in severe pulmonary hypertension. Indian Heart $J$ 2002; 54: 164-169.

11 Kurzyna M, Dabrowsky M, Bielecki D, et al. Atrial septostomy in treatment of end-stage right heart failure in patients with pulmonary hypertension. Chest 2007; 131: 947-948.

12 Sandoval J, Gaspar J, Pulido T, et al. Graded balloon dilation atrial septostomy in severe primary pulmonary hypertension. A therapeutic alternative for patients non-responsive to vasodilator treatment. J Am Coll Cardiol 1998; 32: 297-304.

13 Barst RJ, McGoon M, Torbicki A, et al. Diagnosis and differential assessment of pulmonary arterial hypertension. J Am Coll Cardiol 2004; 43: Suppl. 12, 40S-47S.

14 Sandoval J, Gaspar J. Atrial Septostomy. In: Peacock AJ, Rubin LJ, eds. Pulmonary Circulation. 2nd Edn. London, Edward Arnold Publishers Ltd., 2004; pp. 319-333.

15 Nihill MR, O'Laughlin MP, Mullins CE. Effects of atrial septostomy in patients with terminal cor pulmonale due to pulmonary vascular disease. Cathet Cardiovasc Diagn 1991; 24: 166-172.

16 Micheletti A, Hislop A, Lammers A, et al. Role of atrial septostomy in the treatment of children with pulmonary arterial hypertension. Heart 2006; 92: 969-972.

17 Allcock RJ, O'Sullivan JJ, Corris PA. Atrial septostomy for pulmonary hypertension. Heart 2003; 89: 1344-1347.

18 Hayden AM. Balloon atrial septostomy increases cardiac index and may reduce mortality among pulmonary hypertension patients awaiting lung transplantation. J Transpl Coord 1997; 7: 131-133.

19 Barst RJ. Role of atrial septostomy in the treatment of pulmonary vascular disease. Thorax 2000; 55: 95-96.

20 Sandoval J, Doyle R. Interventional and surgical modalities of treatment for pulmonary arterial hypertension. In: Barst $\mathrm{R}$, ed. Pulmonary Arterial Hypertension: Diagnosis and Evidence-Based Treatment. Chichester, John Wiley \& Sons Ltd., 2008; pp. 147-179. 
21 Rich S, Dodin E, McLaughlin VV. Usefulness of atrial septostomy as a treatment for primary pulmonary hypertension and guidelines for its application. Am J Cardiol 1997; 80: 369-371.

22 Fraisse A, Chetaille P, Amin Z, et al. Use of Amplatzer fenestrated atrial septal defect device in a child with familial pulmonary hypertension. Pediatr Cardiol 2006; 27: 759-762.

23 O'loughlin AJ, Keogh A, Muller DW. Insertion of a fenestrated Amplatzer atrial septostomy device for severe pulmonary hypertension. Heart Lung Circ 2006; 15: 275-277.

24 Prieto LR, Latson LA, Jennings C. Atrial septostomy using a butterfly stent in a patient with severe pulmonary arterial hypertension. Catheter Cardiovasc Interv 2006; 68: 642-647.
25 Lammers AE, Derrick G, Haworth SG, et al. Efficacy and long-term patency of fenestrated Amplatzer devices in children. Catheter Cardiovasc Interv 2007; 70: 578-584.

26 Althoff TF, Knebel F, Panda A, et al. Long-term follow-up of a fenestrated Amplatzer atrial septal occlude in pulmonary arterial hypertension. Chest 2008; 133; 283-285.

27 Troost E, Delcroix M, Gewillig M, et al. A modified technique of stent fenestration of the interatrial septum improves patients with pulmonary hypertension. Catheter Cardiovasc Interv 2009; 73: 173-179.

28 Chin KM, Rubin LJ. Pulmonary arterial hypertension. J Am Coll Cardiol 2008; 51: 1527-1538. 